\title{
Otimização do processo de secagem osmótica na obtenção de produtos secos da manga Tommy Atkins ${ }^{1}$
}

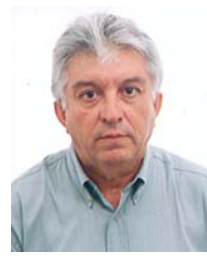

Francisco de A. C. Almeida ${ }^{2}$, Carmelita de F. A. Ribeiro ${ }^{3}$, Satoshi Tobinaga ${ }^{4} \&$ Josivanda P. Gomes ${ }^{2}$

\author{
1 Parte da Dissertação de Mestrado do segundo autor \\ 2 DEAg/CCT/UFCG. Av. Aprígio Veloso, 882, CEP 58100-970. Campina Grande, PB. Fone: (83) 3310-1286. E-mail: \\ diassis@deag.ufcg.edu.br; josi@deag.ufcg.edu.br \\ ${ }^{3}$ Mestre em Engenharia Agrícola. E-mail: carmel amaral@yahoo.com.br \\ ${ }^{4}$ FEA/UNICAMP. Rua Zeferino Vaz, s/n, CEP 13.083, Campinas, SP. Fone: 3788-4028. E-mail: satoshi@fea.unicamp.br
}

Protocolo 110 - 29/7/2004 - Aprovado em 20/7/2005

\begin{abstract}
Resumo: Frutas tropicais como a manga (Mangifera indica L.) uma das mais populares do mundo, embora muito apreciadas, são altamente perecíveis e requer técnicas especiais para sua conservação e comercialização, razão por que se objetivou otimizar seu processo de secagem osmótica e estudar o efeito da temperatura, concentração de sacarose e tempo de imersão, em relação à perda de água e ganho de sólidos. A influência de cada variável e sua interação sobre as respostas, foram avaliada através da análise de variância (teste F e coeficiente de determinação). Verificou-se que os três fatores mostraram efeito significativo quanto à perda de água e ganho de sólidos de manga, dentro das faixas estudadas. A condição ótima encontrada para desidratação utilizada como pré-tratamento para maior perda de água e menor ganho de sólidos, foi temperatura de 50 ${ }^{\circ} \mathrm{C}$, concentração de $40 \%$ e tempo de imersão de 90 min.
\end{abstract}

Palavras-chave: Mangifera indica L., sacarose, desidratação

\section{Optimization of the osmotic drying process to abtain dry products of Tommy Atkins mango}

\begin{abstract}
Tropical fruits like mango (Mangifera indica L.), one of the most popular fruits of the world, although very much appreciated, is highly perishable and seguire special techniques for its conservation and commercialization. This work had as objective to optimize the process of osmotic drying of mango and to study the effect of the temperature, sucrose concentration and time of immersion on the loss of water and gain in solid content. The influence of each variable and its interactions on the response were analyzed through analysis of variance (F-test and determination coefficient). It was verified that the three factors show significant effect on the loss of water and gain of mango solids, within the limits of the study. The optimum condition found for dehydration used as pretreatment for larger loss of water and smaller gain of solids was: temperature of $50{ }^{\circ} \mathrm{C}$, concentration of $40 \%$ and immersion time of $90 \mathrm{~min}$.
\end{abstract}

Key words: Mangifera indica L., sucrose, dehydration

\section{INTRODUÇÃO}

Uma das principais causas da deterioração de alimentos frescos e de conservados é a quantidade de água livre neles presente, fato que, para diversos pesquisadores (El-Aouar, 2001; Azoubel, 2002) deve ser resolvido mediante técnicas de desidratação, para cujo emprego Borges \& Menegalli (1994) informam que a utilização da desidratação osmótica seguida da secagem convectiva, geralmente fornece um produto atrativo ao consumo minimizando, desta forma, os danos causados pelo calor à cor, à textura e ao sabor do alimento.
Um grande avanço na conservação de alimentos é a combinação desses métodos, baseada em tecnologias simples em que se utilizam dois ou mais fatores de conservação, promovendo a estabilidade do alimento, a temperatura ambiente. Para Aguirre \& Gasparino Filho (2001) durante o processo de secagem osmótica os gradientes de atividade de água e de solutos resultam em um fluxo de água através da membrana semipermeável que permite as trocas com o interior, a qual consiste, basicamente, na remoção da água diluída contida dentro da membrana por efeito de uma solução mais concentrada. Ademais, pela natureza da membrana, pode resultar 
no transporte de solutos, particularmente da solução osmótica para a fruta, de modo que a concentração osmótica resulte numa difusão simultânea da água e do soluto.

Para Magalhães \& Queiroz (2001) a desidratação osmótica mostrou-se ser simples quando aplicada em abacaxi, proporcionando a obtenção de um produto com características similares aos produtos in natura. Mediante a aplicação desta técnica, Santos (2003) estudou a elaboração de um estruturado de manga e obteve eficiência na pré-secagem, possibilitando o uso de temperaturas amenas e tempos relativamente curtos, implicando não só em economia de energia mas, principalmente, conferindo à fruta desidratada características mais próximas da fruta in natura.

Argaiz et al. (1994) estudaram a desidratação osmótica de mamão com xarope de milho em calda e avaliaram a concentração osmótica quanto a perda de água e de peso e ganho de sólidos e constataram aumento em ganho de sólidos e decréscimo em perda de água e perda de peso, de 7,3 para $21,1 \%, 76,1$ para $49,1 \%$ e 72,0 para $28,8 \%$, respectivamente. Tedjo et al. (2002) comparando métodos de pré-tratamento e cinéticas de difusão de sólidos em água de mangas desidratadas osmoticamente, viram que o pré-tratamento influenciou na cor e na textura do produto, porém, independendo do tempo de desidratação com maior ganho de sólidos e maior perda de água. Barbosa Júnior (2002) estudou a influência dos fatores temperatura e concentração da solução de cloreto de sódio durante a desidratação osmótica de abóbora e cenoura e concluiu que esses fatores foram de grande influência positiva sobre a perda de água, sobretudo para a abóbora. Em relação ao tema, Farsas \& Lazar (1993) relacionaram, em seu trabalho, a temperatura, como fator de grande influência no processo de desidratação osmótica, por aumentar a taxa de desidratação e o ganho de sólidos, além de modificar as propriedades da solução e do produto.

Pina et al. (2003) estudaram o processamento e a conservação de manga por métodos combinados através de dois experimentos de branqueamento com vapor saturado a $100^{\circ} \mathrm{C}$ por 2 min, na proporção de 1:2 (fruto:xarope) e com concentração de xarope, $25^{\circ}$ Brix; ácido cítrico, $\mathrm{pH}=2,5$; ácido ascórbico, 600 ppm; concentração de benzoato de sódio, 1000 ppm; concentração de cloreto de cálcio, 100 ppm; tratamento térmico, água quente a $100{ }^{\circ} \mathrm{C}$ por 1 min e constataram variações apenas na concentração de $\mathrm{SO}_{2}$, entre 600 a 900 ppm. Após os experimentos, os produtos obtidos foram armazenados durante 120 dias. Os resultados comprovaram que a conservação por métodos combinados promoveu estabilidades microbiológicas e sensoriais da manga em pedaços por, no mínimo, 120 dias, a temperatura de $28^{\circ} \mathrm{C}$. Alves \& Silveira (2002) estudando a secagem de tomates desidratados e não-desidratados osmoticamente, através de secador de bandejas e estufa a vácuo, concluíram que a taxa de desidratação osmótica foi influenciada pela concentração da solução osmótica e a temperatura do processo e, ainda, que esta temperatura exerceu maior influência na cinética da secagem que a velocidade e a pressão a vácuo.

Desta forma objetivou-se, com este trabalho, a otimização do processo de secagem osmótica na obtenção de produtos secos da manga Tommy Atkins.

\section{MATERIAL E MÉTODOS}

Este trabalho foi conduzido no Laboratório de Armazenamento e Processamento de Produtos Agrícolas da Universidade Federal de Campina Grande, PB, e no Laboratório de Medidas Físicas da Faculdade de Engenharia de Alimentos da Universidade Estadual de Campinas, SP.

Foram utilizadas mangas (Mangifera indica L.) no estágio semimaduro da variedade Tommy Atkins, proveniente da região do Vale do São Francisco, BA. Na seleção, considerou-se cor da casca, grau de maturação, tamanho e forma; depois, foram lavadas em água corrente, descascadas e cortadas em placa plana de $0,5 \mathrm{~cm}$ de espessura, utilizando-se faca de aço inoxidável e um cortador planejado para esta finalidade.

Os experimentos de desidratação osmótica foram realizados em uma incubadora (Shaker, marca Tecnal, modelo TE 42) com temperatura e agitação controladas; as mangas, depois de selecionadas, foram pesadas e colocadas em béqueres de 250 $\mathrm{mL}$ contendo solução desidratante; em seguida, os béqueres foram colocados em incubadora e fixados em bandeja agitadora, com controle de temperatura e agitação de $90 \mathrm{rpm}$. A relação amostra/solução de 1:10 nas concentrações e temperaturas apropriadas foi utilizada para diminuir a diluição da solução osmótica. Após o tempo predeterminado, as amostras foram retiradas da incubadora e da solução desidratante, enxaguadas com água destilada para retirada do excesso de solução osmótica, colocadas em papel absorvente, pesadas e embaladas em filme de PVC e levadas a um dessecador para posterior análise de umidade. Com os dados obtidos, avaliou-se o ganho de sólidos e a perda de água, considerando-se a umidade final do produto desidratado.

A umidade das amostras foi determinada retirando-se em torno de $50 \%$ do material de cada placa do produto desidratado, os quais eram colocados em cadinhos de papel alumínio e levados a uma estufa a vácuo (Marca Marconi, MA 030/12) pelo período de $24 \mathrm{~h}$.

Estudou-se a formulação mais apropriada testando-se dois agentes osmóticos em diferentes concentrações, em que na primeira se utilizou a sacarose comercial de marca União e, na segunda, o xarope de milho, de marca Morex 1940 (Corn Products Brasil).

As soluções foram preparadas a concentrações estabelecidas e homogeneizadas em um agitador magnético e depois deixadas em repouso até a sua estabilização (limpas e homogêneas) para serem utilizadas. Os parâmetros perda de peso (PP), perda de água (WL) e ganho de sólidos (GS) foram calculados através das equações abaixo, respectivamente. Os experimentos foram realizados em triplicata.

$$
\begin{gathered}
\operatorname{PP}(\%)=100 \frac{\left(\mathrm{M}_{\mathrm{o}}-\mathrm{M}_{\mathrm{t}}\right)}{\mathrm{M}_{\mathrm{o}}} \\
\mathrm{WL}(\%)=100 \frac{\mathrm{MA}_{\mathrm{O}}-\left(\mathrm{M}_{\mathrm{t}}-\mathrm{MS}_{\mathrm{t}}\right)}{\mathrm{M}_{\mathrm{t}}} \\
\mathrm{GS}(\%)=100 \frac{\left(\mathrm{Ms}_{\mathrm{t}}-\mathrm{Ms}_{\mathrm{O}}\right)}{\mathrm{Mo}}
\end{gathered}
$$


em que:

$\mathrm{M}_{\mathrm{o}}$ - peso inicial da amostra, $\mathrm{g}$

MAo - peso da água, g

$M_{t} \quad$ - peso da amostra, $g$

$\mathrm{MS}_{\mathrm{t}}$ - peso inicial dos sólidos, $\mathrm{g}$

$\mathrm{Ms}_{\mathrm{o}}$ - peso dos sólidos no tempo $\mathrm{t}, \mathrm{g}$

Utilizou-se um planejamento fatorial completo $\left(2^{3}\right)$ com pontos centrais, conforme Barros Neto et al. (2001) observandose os efeitos da temperatura, concentração e tempo sobre a perda de peso, perda de água e ganho de sólidos do processo de desidratação osmótica com a finalidade de se verificar a condição de processo mais favorável à maximização de perda de água e minimização de ganho de sólidos.

Cada tratamento consistiu de 17 ensaios (Tabela 1) sendo os 8 experimentos iniciais do fatorial completo (modelo linear), três no ponto central e seis para a configuração estrela (modelo quadrático). As variáveis dependentes são: perda de peso, perda de água e ganho de sólidos, que são função das variáveis

Tabela 1. Matriz do planejamento codificada

\begin{tabular}{cccc}
\hline Ensaio & Temperatura $\left({ }^{\circ} \mathrm{C}\right)$ & Concentração (\%) & Tempo (min) \\
\hline 1 & -1 & -1 & -1 \\
2 & 1 & -1 & -1 \\
3 & -1 & 1 & -1 \\
4 & 1 & 1 & -1 \\
5 & -1 & -1 & 1 \\
6 & 1 & -1 & 1 \\
7 & -1 & 1 & 1 \\
8 & 1 & 1 & 1 \\
9 & $-1,68$ & 0 & 0 \\
10 & $+1,68$ & 0 & 0 \\
11 & 0 & $-1,68$ & 0 \\
12 & 0 & $+1,68$ & 0 \\
13 & 0 & 0 & $-1,68$ \\
14 & 0 & 0 & $+1,68$ \\
15 & 0 & 0 & 0 \\
16 & 0 & 0 & 0 \\
17 & 0 & 0 & 0 \\
\hline
\end{tabular}

independentes: temperatura, concentração da solução e tempo de imersão.

A escolha das faixas experimentais utilizadas no estudo baseou-se em resultados obtidos a partir de ensaios preliminares (Tabela 2).

Tabela 2. Níveis reais e codificados das variáveis independentes no planejamento fatorial

\begin{tabular}{lccccc}
\hline Fatores & \multicolumn{5}{c}{ Níveis } \\
\cline { 2 - 6 } Variáveis & $-\alpha$ & -1 & 0 & +1 & $+\alpha$ \\
\hline Temperatura $\left({ }^{\circ} \mathrm{C}\right)$ & 30 & 34 & 40 & 46 & 50 \\
Concentração da solução (\%) & 40 & 44 & 50 & 56 & 60 \\
Tempo (min) & 90 & 120 & 165 & 210 & 240 \\
\hline
\end{tabular}

Os ajustes dos dados experimentais aos modelos de desidratação osmótica foram realizados por meio de regressão linear e não-linear utilizando-se do programa Statistica versão 5.0. Através da análise de variância (ANOVA), do teste de Fischer (F) e do coeficiente de correlação, realizaram-se os estudos de significância estatística do planejamento fatorial. Para a escolha dos melhores ajustes, utilizaram-se como critérios, o percentual de variação explicada (Barros Neto et al., 2001) e o módulo do desvio relativo médio (P) definido como a média da percentagem da diferença relativa entre os valores experimentais e preditos (Lomauro et al., 1985).

\section{RESULTADOS E DISCUSSÃO}

Os resultados da desidratação osmótica, de acordo com o planejamento fatorial proposto, encontram-se na Tabela 3, para as variáveis de respostas: perda de peso (PP), perda de água (WL) e ganho de sólidos (GS).

Em análises aos dados da Tabela 3, tem-se maior perda de peso e de água e menor ganho de sólidos para a desidratação realizada com o xarope de milho. As exceções se referem às

Tabela 3. Perda de peso, perda de água e ganho de sólidos no processo de desidratação osmótica da manga Tommy Atkins em soluções de sacarose e xarope de milho

\begin{tabular}{|c|c|c|c|c|c|c|c|c|c|}
\hline \multirow{2}{*}{ Ensaio } & \multirow{2}{*}{$\mathrm{T}\left({ }^{\circ} \mathrm{C}\right)$} & \multirow{2}{*}{$\mathrm{C}(\%)$} & \multirow{2}{*}{$\mathrm{T}(\min )$} & \multicolumn{2}{|c|}{ PP $(\%)$} & \multicolumn{2}{|c|}{ WL $(\%)$} & \multicolumn{2}{|c|}{ GS $(\%)$} \\
\hline & & & & Sacarose & Xarope de Milho & Sacarose & Xarope de Milho & Sacarose & Xarope de Milho \\
\hline 1 & 34 & 44 & 120 & 15,318 & 16,938 & 26,760 & 25,641 & 11,442 & 8,704 \\
\hline 2 & 46 & 44 & 120 & 33,318 & 32,090 & 41,847 & 39,986 & 8,529 & 7,896 \\
\hline 3 & 34 & 56 & 120 & 33,294 & 21,386 & 39,696 & 31,080 & 6,403 & 9,694 \\
\hline 4 & 46 & 56 & 120 & 34,838 & 41,355 & 43,156 & 43,595 & 7,748 & 2,240 \\
\hline 5 & 34 & 44 & 210 & 21,179 & 32,972 & 34,888 & 40,685 & 13,709 & 7,712 \\
\hline 6 & 46 & 44 & 210 & 34,999 & 41,247 & 42,863 & 46,976 & 7,864 & 5,729 \\
\hline 7 & 34 & 56 & 210 & 34,950 & 46,796 & 43,943 & 51,815 & 8,993 & 5,019 \\
\hline 8 & 46 & 56 & 210 & 44,059 & 47,645 & 52,089 & 51,394 & 8,031 & 3,750 \\
\hline 9 & 30 & 50 & 165 & 22,389 & 36,954 & 33,123 & 39,039 & 10,734 & 2,085 \\
\hline 10 & 50 & 50 & 165 & 28,310 & 42,247 & 40,313 & 48,258 & 12,003 & 6,011 \\
\hline 11 & 40 & 40 & 165 & 32,048 & 26,669 & 39,879 & 29,782 & 7,831 & 3,113 \\
\hline 12 & 40 & 60 & 165 & 47,995 & 52,992 & 54,326 & 54,757 & 6,331 & 1,765 \\
\hline 13 & 40 & 50 & 90 & 30,641 & 25,648 & 36,760 & 31,187 & 6,119 & 5,538 \\
\hline 14 & 40 & 50 & 240 & 21,961 & 51,690 & 36,137 & 53,651 & 14,176 & 1,962 \\
\hline 15 & 40 & 50 & 165 & 14,916 & 43,544 & 28,894 & 46,091 & 13,977 & 2,547 \\
\hline 16 & 40 & 50 & 165 & 15,064 & 44,967 & 28,991 & 47,352 & 13,928 & 2,384 \\
\hline 17 & 40 & 50 & 165 & 13,516 & 43,908 & 27,966 & 46,620 & 14,450 & 2,712 \\
\hline
\end{tabular}

PP - Perda de Peso; WL - Perda de Água; GS - Ganho de Sólidos 
amostras 2, 3, 11 e 13, em que a perda de peso foi menor em solução de xarope de milho e, com relação ao ganho de sólidos (GS), apenas na amostra 3, este foi maior com a solução de sacarose. Observa-se, para a solução de xarope de milho, menor incorporação de sólidos e conseqüentemente, mais rápida a saída de água, o que está de acordo com Contreras \& Smyrl (1981) que afirmam que o coeficiente de difusividade da água depende do conteúdo de sólidos dissolvidos, ou seja, a difusividade decresce com o aumento de sólidos e que, com o aumento da temperatura, foram maiores o ganho de sólidos e a perda de água, porém menor o tempo de processo. Esses resultados concordam com os obtidos por Rastogi \& Raghavarao (1994) que estudaram o efeito da concentração e temperatura sobre a desidratação osmótica de coco, constatando que a elevação da temperatura de 24 a $45^{\circ} \mathrm{C}$ e a concentração de sacarose de 40 a $70{ }^{\circ} \mathrm{Brix}$, proporcionavam aumento no ganho de sólidos e perda de água; referidas observações também se apóiam na pesquisa de Argaiz et al. (1994) que, estudando a desidratação osmótica de mamão concluíram que a concentração da solução osmótica de xarope de milho foi fator de grande influência neste processo.

\section{Perda de peso}

O resumo da análise estatística aplicada aos dados experimentais de perda de peso (PP) na desidratação osmótica da manga Tommy Atkins em solução de sacarose e xarope de milho encontra-se contido na Tabela 4, na qual se observam os efeitos significativos a $5 \%$ de probabilidade dos fatores lineares, quadráticos e suas interações; ademais, encontra-se também, o efeito não significativo desses fatores.

$\mathrm{O}$ valor dos efeitos estimados indica o quanto cada fator (temperatura, concentração e tempo) influi na resposta observada, neste caso a perda de peso da manga Tommy Atkins durante o processo de desidratação osmótica. Quando positivo indica que, ao passar de um valor mínimo a um valor máximo da variável, a resposta aumenta e, quando negativo, dá-se o contrário. A perda de peso em solução de sacarose não foi significativa a $5 \%$ de probabilidade para os fatores tempo (L) e as interações temperatura $\mathrm{x}$ tempo e concentração $\mathrm{x}$ tempo. Tem-se, ainda, que os efeitos significativos, à exceção da interação temperatura x concentração para efeito estimado, são todos positivos, indicando que o aumento desses fatores exerce aumento na perda de peso e, também, que a concentração foi o fator que exerceu maior influência no processo de desidratação osmótica da manga com o emprego de sacarose, seguido da temperatura revelada pelo efeito estimado, comportamento que o erro puro não revelou, uma vez que os valores desses fatores são de mesma amplitude. Para a desidratação osmótica em soluções de xarope de milho, os efeitos estimados das interações temperatura $\mathrm{x}$ concentração e concentração $\mathrm{x}$ tempo não são significativos a $5 \%$ de probabilidade, mas são desprezados do modelo de predição.

Para os fatores significativos temperatura, concentração e tempo em termos lineares, os efeitos são positivos sobre a perda de peso, ou seja, um aumento em qualquer um desses fatores acarreta aumento na perda de peso, enquanto os efeitos em termos quadráticos para os mesmos fatores e mais a interação temperatura $x$ tempo, são de influência negativa, em que um aumento nesses fatores vai acarretar diminuição na perda de peso. Da análise, verifica-se que, com a utilização da sacarose como agente osmótico, obtiveram-se mais efeitos positivos sobre a perda de peso para a desidratação osmótica de manga que a solução de xarope de milho.

Ao longo do tempo de processamento, a água tende a uma estabilização ou redução devido, provavelmente, ao equilíbrio atingido com o soluto do meio. Heng et al. (1990) observaram que a medida em que se aumenta a massa molar do soluto, temse diminuição dos sólidos incorporados e aumento da perda de água favorecendo, desta forma, a perda de peso. Depois da eliminação dos fatores não significativos foram realizadas a análise de variância (ANOVA) e a significância de regressão, a 95\% de confiança, utilizando-se o teste de Fisher (F) conforme a Tabela 5 .

Verificou-se que os modelos apresentaram regressão e falta de ajuste significativo a $5 \%$ de probabilidade, com $\mathrm{F}$ calculado de 5,59 e 1,26 vezes maior que o $F$ tabelado, respectivamente. Segundo Barros Neto et al. (2001) embora uma regressão seja significativa através do teste $\mathrm{F}$, o modelo pode não ser preditivo, ou seja, ele pode não servir para realizar previsões a partir do modelo gerado, por cobrir uma variação pequena dos fatores estudados; então, para que uma regressão seja considerada preditiva, o valor de $\mathrm{F}$ calculado deve ser no mínimo 4 a 5 vezes o valor do $F$ tabelado (Box \& Wetz, 1973).

$\mathrm{O}$ coeficiente de correlação $\left(\mathrm{R}^{2}\right)$ obtido para os modelos ajustados foram superiores a 0,90 , o que significa dizer que a percentagem de variação explicada pelo modelo foi maior que $90 \%$; desta forma, os modelos ajustados foram estatisticamente significativos. De acordo com Barros Neto et al. (2001) na

Tabela 4. Efeito estimado, erro puro e significância estatística, para cada fator no modelo codificado para perda de peso em soluções de sacarose e xarope de milho

\begin{tabular}{|c|c|c|c|c|c|c|}
\hline \multirow{3}{*}{$\begin{array}{c}\text { Fatores } \\
\text { Média Global }\end{array}$} & \multicolumn{2}{|c|}{ Efeito Estimado } & \multicolumn{2}{|c|}{ Erro Puro } & \multicolumn{2}{|c|}{ Significância Estatística } \\
\hline & Sacarose & Xarope de Milho & Sacarose & Xarope de Milho & Sacarose & Xarope de Milho \\
\hline & 14,50 & 44,40 & 0,49 & 0,42 & 0,00 & 0,00 \\
\hline Temperatura (Q) & 7,65 & $-5,00$ & 0,50 & 0,44 & 0,00 & 0,00 \\
\hline Concentração (L) & 10,13 & 11,45 & 0,46 & 0,40 & 0,00 & 0,00 \\
\hline Concentração (Q) & 18,05 & $-4,84$ & 0,50 & 0,44 & 0,00 & 0,00 \\
\hline Temperatura x Concentração & $-5,29$ & $-0,65^{\mathrm{ns}}$ & 0,60 & $0,52^{\mathrm{ns}}$ & 0,12 & $0,33^{\mathrm{ns}}$ \\
\hline Temperatura $\mathrm{x}$ Tempo & $0,84^{\mathrm{ns}}$ & $-6,49$ & $0,60^{\mathrm{ns}}$ & 0,52 & $0,29^{\mathrm{ns}}$ & 0,00 \\
\hline Concentração x Tempo & $0,83^{\mathrm{ns}}$ & $1,62^{\mathrm{ns}}$ & $0,60^{\mathrm{ns}}$ & $0,52^{\mathrm{ns}}$ & $0,30^{\mathrm{ns}}$ & $0,89^{\mathrm{ns}}$ \\
\hline
\end{tabular}


Tabela 5. Resultados da análise de variância para o modelo ajustado para perda de peso na desidratação osmótica da manga Tommy Atkins em soluções de sacarose e xarope de milho

\begin{tabular}{|c|c|c|c|c|c|}
\hline \multirow{2}{*}{ FV } & GL & SQ & QM & $\mathrm{F}_{\text {calculado }}$ & $\mathrm{F}_{\text {tabelado }}$ \\
\hline & \multicolumn{5}{|c|}{ Sacarose } \\
\hline Regressão & 6 & 1560,422 & 260,07 & 18,03 & 3,22 \\
\hline Resíduo & 10 & 144,237 & 14,423 & & \\
\hline $\mathrm{Fa}$ & 8 & 142,778 & 17,847 & 24,48 & 19,37 \\
\hline Erro puro & 2 & 1,459 & 0,729 & & \\
\hline Total & 16 & 1704,659 & & $\mathrm{R}^{2}=0,91$ & \\
\hline FV & \multicolumn{5}{|c|}{ Xarope de Milho } \\
\hline Regressão & 7 & 1624,948 & 232,13 & 12,07 & 3,29 \\
\hline Resíduo & 9 & 173,005 & 19,22 & & \\
\hline $\mathrm{Fa}$ & 7 & 171,912 & 24,55 & 44,96 & 19,35 \\
\hline Erro puro & 2 & 1,093 & 0,546 & & \\
\hline Total & 16 & 1797,953 & & $\mathrm{R}^{2}=0,90$ & \\
\hline
\end{tabular}

análise de variância de um modelo uma parte da variação total das observações em torno da média é descrita pela equação de regressão, enquanto o restante faz parte dos resíduos; assim, quanto maior for a fração descrita pela regressão, ou melhor, quanto mais próximo de 1 for o valor de $\mathrm{R}^{2}$, melhor será o ajuste do modelo aos dados observados.

Para a perda de peso do processo de desidratação osmótica em xarope de milho, os modelos apresentaram regressão e falta de ajuste significativo a $5 \%$ de probabilidade, com $\mathrm{F}$ calculado maior que o $\mathrm{F}$ tabelado. As equações representativas do modelo ajustado para descrever a perda de peso (PP) na desidratação osmótica da manga Tommy Atkins em soluções de sacarose e xarope de milho, encontram-se Eqs. 4 e 5, em que: $\mathrm{T}$ - temperatura $\left({ }^{\circ} \mathrm{C}\right) ; \mathrm{C}$ - concentração $(\%)$ e $\mathrm{t}$ - tempo (min).

$$
\begin{gathered}
\mathrm{PP}_{(\mathrm{a})}(\%)=14,50+3,84 \mathrm{~T}+3,83 \mathrm{~T}^{2}+5,06 \mathrm{C}+ \\
9,03 \mathrm{C}^{2}+4,16 \mathrm{t}^{2}-2,64 \mathrm{TC} \\
\mathrm{PP}_{(\mathrm{b})}(\%)=44,41+3,89 \mathrm{~T}-2,50 \mathrm{~T}^{2}+5,73 \mathrm{C}- \\
2,42 \mathrm{C}^{2}+7,37 \mathrm{t}-2,83 \mathrm{t}^{2}-3,25 \mathrm{Tt}
\end{gathered}
$$

Como se vê, a perda de peso no processo de desidratação osmótica em solução de sacarose aumentou com o aumento da concentração. Maiores valores de perda de peso podem ser obtidos utilizando-se concentrações acima de $58 \%$ e temperaturas entre 48 a $50{ }^{\circ} \mathrm{C}$, independente do tempo de processo. Observa-se ainda que, independente do tempo, o maior valor de perda de peso pode ser obtido na temperatura de $50{ }^{\circ} \mathrm{C}$ e concentração de $60 \%$. Para a manga desidratada em solução de xarope de milho, verifica-se que o tempo de processo foi o fator de maior influência. Maiores valores de perda de peso podem ser obtidos com tempos superiores a $130 \mathrm{~min}$, concentrações acima $45 \%$ e temperaturas acima de $38^{\circ} \mathrm{C}$.

Argaiz et al. (1994) constataram, na desidratação osmótica do mamão, aumento no ganho de sólidos e decréscimo na perda de peso, o que se harmoniza com os resultados deste trabalho, para tal fator (perda de peso). Este fato se deve, no presente trabalho, ao gradiente de atividade de água da sacarose (solução) e do xarope de milho, que resultou em um fluxo de água através da membrana semipermeável da manga, o que explica Aguirre \& Gasparino Filho (2001) ao afirmarem que, pela natureza da membrana, pode resultar no transporte de solutos, particularmente da solução osmótica para a fruta, de modo que a concentração osmótica resulta numa difusão simultânea de água e soluto.

\section{Perda de água}

Os efeitos dos fatores lineares, quadráticos e da interação para a perda de água em soluções de sacarose e xarope de milho, estão representados na Tabela 6 .

Os valores dos efeitos estimados indicam o quanto cada fator influi na resposta observada, ou seja, quanto maior o valor dos efeitos maior também será a influência sobre a perda de água. Para a desidratação osmótica de manga Tommy Atkins em solução de sacarose, observa-se que os fatores temperatura, concentração e tempo lineares e quadráticos, além da interação temperatura x concentração, são significativos a $5 \%$ de probabilidade. A perda de água durante o processo de desidratação será mais intensa com o aumento desses fatores lineares e quadráticos, ao contrário da interação temperatura $x$ concentração, que tem influência negativa, de forma inversa, em que um aumento acarreta diminuição na perda de água. As

\begin{tabular}{|c|c|c|c|c|c|c|}
\hline \multirow{3}{*}{$\begin{array}{c}\text { Fatores } \\
\text { Média Global }\end{array}$} & \multicolumn{2}{|c|}{ Efeito Estimado } & \multicolumn{2}{|c|}{ Erro Puro } & \multicolumn{2}{|c|}{ Significância Estatística } \\
\hline & Sacarose & Xarope de Milho & Sacarose & Xarope de Milho & Sacarose & Xarope de Milho \\
\hline & 28,62 & 46,76 & 0,32 & 0,36 & 0,00 & 0,00 \\
\hline Temperatura (L) & 6,85 & 7,06 & 0,30 & 0,34 & 0,00 & 0,00 \\
\hline Temperatura (Q) & 5,67 & $-2,66$ & 0,33 & 0,37 & 0,00 & 0,01 \\
\hline Concentração (L) & 8,32 & 9,75 & 0,30 & 0,34 & 0,00 & 0,00 \\
\hline Concentração (Q) & 13,02 & $-3,63$ & 0,33 & 0,37 & 0,00 & 0,01 \\
\hline Tempo (L) & 3,11 & 12,94 & 0,30 & 0,34 & 0,00 & 0,00 \\
\hline Tempo (Q) & 5,47 & $-3,53$ & 0,33 & 0,37 & 0,00 & 0,01 \\
\hline Temperatura x Concentração & $-2,86$ & $-2,13$ & 0,40 & 0,44 & 0,01 & 0,04 \\
\hline Temperatura $\mathrm{x}$ Tempo & $-0,60^{\mathrm{ns}}$ & $-5,24$ & $0,40^{\mathrm{ns}}$ & 0,44 & $0,26^{\mathrm{ns}}$ & 0,00 \\
\hline Concentração x Tempo & $1,00^{\mathrm{ns}}$ & $1,62^{\mathrm{ns}}$ & $0,40^{\mathrm{ns}}$ & $0,44^{\mathrm{ns}}$ & $0,12^{\mathrm{ns}}$ & $0,68^{\mathrm{ns}}$ \\
\hline
\end{tabular}
interações temperatura $\mathrm{x}$ tempo e concentração $\mathrm{x}$ tempo não

Tabela 6. Efeito estimado, erro puro e grau de significância estatística, para cada fator no modelo codificado para perda de água em soluções de sacarose e xarope de milho

ns - não significativos; (L) - Termo Linear; (Q) - Termo Quadrático 
foram significativas e por isso não serão utilizadas no modelo de predição. Os fatores concentração e temperatura foram os efeitos de maior influência sobre a perda de água.

Observa-se, para a desidratação osmótica, quando é utilizado o xarope de milho, que quase todos os efeitos dos fatores, com exceção da interação concentração x tempo, são significativos a $5 \%$ de probabilidade. Os fatores temperatura, concentração e tempo, em termos lineares, obtiveram efeito positivo sobre a perda de água, indicando que se forem elevadas temperatura, concentração e tempo de imersão, maior será a perda de água. Esses mesmos fatores, em termos quadráticos, e as interações temperatura x concentração e temperatura $x$ tempo, têm influência negativa sobre a perda de água, indicando que o aumento nesses fatores acarreta diminuição na perda de água. $\mathrm{O}$ fator tempo (L) foi o que exerceu maior influência para a perda de água na desidratação osmótica em xarope de milho. Verifica-se, para a desidratação com xarope de milho, que os efeitos dos fatores temperatura, concentração e tempo (termos lineares) foram superiores quando comparados com os de sacarose, porém em termos quadráticos a sacarose foi superior.

Na Tabela 7 encontram-se os resultados da análise de variância para o modelo ajustado de perda de água na desidratação osmótica em solução de sacarose e xarope de milho.

Tabela 7. Resultados da ANOVA para o modelo ajustado de perda de água na desidratação osmótica da manga em solução de sacarose xarope e de milho

\begin{tabular}{|c|c|c|c|c|c|}
\hline \multirow{2}{*}{ FV } & GL & SQ & QM & $\mathrm{F}_{\text {calculado }}$ & $\mathrm{F}_{\text {tabelado }}$ \\
\hline & \multicolumn{5}{|c|}{ Sacarose } \\
\hline Regressão & 7 & 938,573 & 134,08 & 18,20 & 3,29 \\
\hline Resíduo & 9 & 66,304 & 7,367 & & \\
\hline $\mathrm{Fa}$ & 7 & 65,664 & 9,380 & 29,31 & 19,35 \\
\hline Erro puro & 2 & 0,640 & 0,32 & & \\
\hline Total & 16 & 1004,880 & \multicolumn{3}{|c|}{$\mathrm{R}^{2}=0,90$} \\
\hline FV & \multicolumn{5}{|c|}{ Xarope de Milho } \\
\hline Regressão & 8 & 1190,019 & 148,75 & 14,42 & 3,44 \\
\hline Resíduo & 8 & 82,497 & 10,31 & & \\
\hline $\mathrm{Fa}$ & 6 & 81,696 & 13,61 & 33,98 & 19,33 \\
\hline Erro puro & 2 & 0,801 & 0,4005 & & \\
\hline Total & 16 & 1272,516 & \multicolumn{3}{|c|}{$\mathrm{R}^{2}=0,93$} \\
\hline
\end{tabular}

Nota-se que o xarope de milho não foi tão bem sucedido quando comparado com a sacarose para todos os fatores estudados (Tabela 7), já que quase todos tiveram influência negativa, sobre a perda de água. Após a eliminação dos parâmetros não significativos realizaram-se a análise de variância e o teste de Fisher (F), para a perda de água no processo de desidratação osmótica de manga em soluções de sacarose e xarope de milho.

Para a desidratação osmótica de manga em solução de sacarose, verificou-se que o modelo apresentou regressão e falta de ajuste significativo a $5 \%$ de probabilidade, com $\mathrm{F}$ calculado maior que o $\mathrm{F}$ tabelado. O modelo ajustado apresentou regressão e falta de ajuste com $\mathrm{F}$ calculado de 5,53 e 1,51 vezes maior que o $F$ tabelado, respectivamente, sendo o modelo considerado preditivo; igualmente, para a solução em xarope de milho, $F$ calculado em 4,19 e 1,75 vezes maior que o $F$ tabelado.

Os modelos propostos para descrever a perda de água na desidratação osmótica da manga em soluções de sacarose (a) e xarope de milho (b), estão de acordo com as Eq. 6 e 7, em que: T é a temperatura, ${ }^{\circ} \mathrm{C} ; \mathrm{C}$ é a concentração, $\%$ e té o tempo, min.

$$
\begin{gathered}
\mathrm{WL}_{(\mathrm{a})}(\%)=28,63+3,42 \mathrm{~T}+2,84 \mathrm{~T}^{2}+4,16 \mathrm{C}+6,51 \mathrm{C}^{2} \\
+1,559 \mathrm{t}+2,74 \mathrm{t}^{2}-1,43 \mathrm{TC}
\end{gathered}
$$

A concentração foi o fator de maior influência sobre a perda de água na desidratação osmótica da manga em solução de sacarose, enquanto maiores valores de perda de água podem ser obtidos em regiões acima de $58 \%$ de concentração, temperatura superior a $50{ }^{\circ} \mathrm{C}$ e tempos de processo acima de 200 min. Esses resultados estão em conformidade com os obtidos por Silveira et al. (1996) que, estudando a cinética de desidratação de abacaxi, concluíram que a perda de água e o ganho de sólidos aumentam com o aumento da concentração da solução de sacarose. Segundo Moy et al. (1978) a perda de água de um produto submetido a desidratação osmótica está diretamente ligada à concentração da solução e ao tipo de soluto, ou seja, aumentando a concentração da solução osmótica a perda de água será mais favorecida que o ganho de sólidos.

Para a manga desidratada em soluções de xarope de milho tem-se maiores perdas de água para as concentrações acima de $54 \%$, temperaturas superiores a $48^{\circ} \mathrm{C}$ e tempo de imersão maior que $160 \mathrm{~min}$, resultados esses concordantes com os de Azoubel (2002) que, estudando a influência de pré-tratamentos na obtenção de produtos secos de caju, verificou que maiores valores de perda de água poderiam ser obtidos utilizando-se altas concentrações de solução de xarope de milho, em torno de $56 \%$ em 160 min de processo.

\section{Ganho de sólidos}

A Tabela 8 apresenta os efeitos dos fatores lineares, quadráticos e da interação para ganho de sólidos em soluções de sacarose e xarope de milho. Os valores dos efeitos estimados indicam o quanto cada fator influi na resposta de interesse, neste caso, o ganho de sólidos.

Para a sacarose, vê-se que quase todos os efeitos, com exceção da interação concentração $x$ tempo, são significativos a $5 \%$ de probabilidade e, sendo assim, a interação citada será desprezada do modelo codificado, porém, dos efeitos significativos apenas o fator tempo (L) e a interação temperatura $x$ concentração apresentaram efeito de influência positiva sobre o ganho de sólidos, em que um aumento desses fatores influi no aumento de ganho de sólidos.

Para a desidratação em xarope de milho verifica-se que, assim como para a sacarose, a interação concentração $x$ tempo foi à única que não foi significativa a 5\% de probabilidade e, 
Tabela 8. Efeito estimado, erro puro e significância estatística para cada fator no modelo codificado para ganho de sólidos em soluções de sacarose e xarope de milho

\begin{tabular}{|c|c|c|c|c|c|c|}
\hline \multirow{2}{*}{$\begin{array}{c}\text { Fatores } \\
\text { Média Global }\end{array}$} & \multicolumn{2}{|c|}{ Efeito Estimado } & \multicolumn{2}{|c|}{ Erro Puro } & \multicolumn{2}{|c|}{ Significância Estatística } \\
\hline & Sacarose & Xarope de Milho & Sacarose & Xarope de Milho & Sacarose & Xarope de Milho \\
\hline Temperatura (L) & 14,12 & 2,35 & 0,16 & 0,09 & 0,00 & 0,00 \\
\hline Concentração (L) & $-2,01$ & 2,34 & 0,17 & 0,09 & 0,00 & 0,00 \\
\hline Concentração (Q) & $-1,88$ & $-1,70$ & 0,15 & 0,08 & 0,00 & 0,00 \\
\hline Tempo (L) & $-5,05$ & 1,20 & 0,17 & 0,09 & 0,00 & 0,00 \\
\hline Temperatura x Concentração & $-2,88$ & 2,13 & 0,17 & 0,09 & 0,00 & 0,00 \\
\hline Temperatura x Tempo & 2,28 & $-1,48$ & 0,20 & 0,11 & 0,00 & 0,00 \\
\hline Concentração x Tempo & $-1,30^{\mathrm{ns}}$ & $1,25^{\mathrm{ns}}$ & $0,20^{\mathrm{ns}}$ & $0,11^{\mathrm{ns}}$ & $0,02^{\mathrm{ns}}$ & $0,00^{\mathrm{ns}}$ \\
\hline
\end{tabular}

por isso mesmo, desprezada do modelo. Analisando-se os valores dos efeitos significativos, tem-se que os fatores temperatura, concentração e tempo em termos quadráticos e a interação temperatura $\mathrm{x}$ tempo, obtiveram efeito positivo sobre a resposta de interesse, ou seja, um aumento em qualquer um desses fatores significará maior ganho de sólidos enquanto os demais efeitos significativos foram de influência negativa.

Mediante os efeitos estimados para as soluções osmóticas, sacarose e xarope de milho, em relação às variáveis estudadas e suas interações sobre o ganho de sólidos no processo de desidratação osmótica de manga Tommy Atkins, observa-se, para as soluções osmóticas mais efeitos de influência negativa que positiva sobre o ganho de sólidos no processo, sendo a concentração de sacarose o efeito de maior influência negativa para a resposta de interesse.

Após a eliminação dos parâmetros não significativos realizaram-se a análise de variância (ANOVA) e o teste de Fisher (F) para ganho de sólidos no processo de desidratação osmótica de manga Tommy Atkins em solução de sacarose. Observa-se na Tabela 9 que o F calculado para regressão foi de 1,82 vezes maior que o $\mathrm{F}$ tabelado, mas significativo a $5 \%$ de probabilidade; no entanto, admitindo-se que o F calculado deve ser 4 a 5 vezes maior que o tabelado, neste caso o modelo não é considerado preditivo.

O resíduo obteve valor baixo de percentual de variação explicada de apenas $86 \%$ da variação das respostas observadas. Para a desidratação de manga em solução de xarope de milho, o modelo para regressão não foi preditivo com $\mathrm{F}$ calculado menor que $\mathrm{F}$ tabelado, o que confere também
Tabela 9. Resultados da ANOVA para o modelo ajustado para ganho de sólidos na desidratação osmótica da manga em solução de sacarose e xarope de milho

\begin{tabular}{lcrccc}
\hline \multirow{5}{*}{ FV } & GL & SQ & QM & $F_{\text {calculado }}$ & $\mathrm{F}_{\text {tabelado }}$ \\
\cline { 2 - 6 } & \multicolumn{5}{c}{ Sacarose } \\
\hline Regressão & 8 & 130,633 & 16,329 & 6,28 & 3,44 \\
Resíduo & 8 & 20,7842 & 2,5980 & & \\
Fa & 6 & 20,6182 & 3,436 & 41,39 & 19,33 \\
Erro puro & 2 & 0,1660 & 0,083 & & \\
Total & 16 & 151,4181 & & $\mathrm{R}^{2}=0,86$ & \\
\hline
\end{tabular}

\begin{tabular}{lcrccc}
\hline FV & \multicolumn{5}{c}{ Xarope de Milho } \\
\hline Regressão & 8 & 52,3326 & 6,54 & 1,01 & 3,44 \\
Resíduo & 8 & 57,8152 & 6,47 & & \\
Fa & 6 & 57,7616 & 9,62 & 370,00 & 19,33 \\
Erro puro & 2 & 0,0536 & 0,026 & & \\
Total & 16 & 110,1478 & \multicolumn{4}{c}{$\mathrm{R}^{2}=0,47$} \\
\hline FV-Fonte deVariação; GL - Grau de Liberdade; & SQ - Soma Quadrática; MQ - Média Quadrática;
\end{tabular}

FV-Fonte deVariação; GL - Grau de Liberdade; SQ - Soma Quadrática; MQ - Média Quadrática; Fa - Falta deAjuste

valor do resíduo bastante alto e coeficiente de regressão explicando apenas $47 \%$ das respostas observadas.

\section{Otimização do processo de desidratação osmótica}

As razões de ganho de sólidos sobre a perda de água (GS/ WL) para a manga desidratada osmoticamente em soluções de sacarose e xarope de milho de acordo com o planejamento fatorial, podem ser observadas na Tabela 10 .

Conforme se observa, a maximização da perda de água provoca aumento na incorporação de sólidos; em contrapartida,

Tabela 10. Valores da razão, ganho de sólidos (GS) e perda de água (WL), para a desidratação osmótica de manga Tommy Atkins

\begin{tabular}{cccccc}
\hline Ensaios & $\begin{array}{c}\text { Sacarose } \\
\text { GS/WL }\end{array}$ & $\begin{array}{c}\text { Xarope de Milho } \\
\text { GS/WL }\end{array}$ & Ensaios & $\begin{array}{c}\text { Sacarose } \\
\text { GS/WL }\end{array}$ & $\begin{array}{c}\text { Xarope de Milho } \\
\text { GS/WL }\end{array}$ \\
\hline 1 & 0,43 & 0,34 & 10 & 0,30 & 0,12 \\
2 & 0,20 & 0,20 & 11 & 0,20 & 0,10 \\
3 & 0,16 & 0,31 & 12 & 0,12 & 0,03 \\
4 & 0,18 & 0,05 & 13 & 0,17 & 0,18 \\
5 & 0,39 & 0,19 & 14 & 0,39 & 0,06 \\
6 & 0,18 & 0,12 & 15 & 0,48 & 0,05 \\
7 & 0,20 & 0,10 & 16 & 0,48 & 0,06 \\
8 & 0,15 & 0,07 & 17 & 0,52 & \\
9 & 0,32 & 0,05 & & & \\
\hline
\end{tabular}


a minimização da incorporação de sólidos conduz baixos valores de perda de água. A solução concentrada utilizada no processo de desidratação osmótica pode afetar fortemente as características físicas, químicas e biológicas, além de alterar o sabor da fruta, devido à incorporação de sólidos no decorrer do processo. Segundo Raoult-Wack et al. (1994) a incorporação de sólidos e a perda de água são influenciadas pelas características da matéria-prima, como membrana celular, grau de compactação e estrutura do tecido, quantidade de sólidos insolúveis e os espaços intracelulares, fato de grande importância para o processo de desidratação osmótica.

Observa-se, ainda, mediante os dados da Tabela 10, que dentro da faixa experimental estudada de temperatura de 34 a $50{ }^{\circ} \mathrm{C}$, concentração de 40 a $60 \%$ e tempo de imersão de 90 a 240 min para o processo de desidratação osmótica em solução de sacarose, as condições otimizadas no processo, isto é, as condições em que o produto perde mais água e adquire menos sólido, requerem uso de temperaturas em torno de 48 a $50^{\circ} \mathrm{C}$ e concentrações de $40 \%$ com duração de 90 a 120 min. Em relação à otimização do processo de desidratação osmótica em solução de xarope de milho, o produto perde mais água e ganha menos sólidos em temperaturas de 38 a $48{ }^{\circ} \mathrm{C}$, concentrações de 50 a $60 \%$ e tempos intermediários de 160 a 220 min.

Com a finalidade de se obter maior perda de água e menor ganho de sólidos e encontrar a melhor condição para validação do método combinado mantendo-se o máximo possível as características iniciais do produto, encontraram-se duas condições ótimas de processo: para sacarose a $\mathrm{T}=50^{\circ} \mathrm{C}$ e $\mathrm{C}=$ $40 \%$ e T $=90 \mathrm{~min}$; e para o xarope de milho: $\mathrm{T}=40^{\circ} \mathrm{C}$ e $\mathrm{C}=50 \%$ $\mathrm{e} \mathrm{T}=160 \mathrm{~min}$.

\section{CONCLUSÕES}

1. A perda de peso, perda de água e o ganho de sólidos aumentaram com o aumento das variáveis: temperatura, concentração e tempo de imersão, durante o processo de desidratação osmótica de manga Tommy Atkins.

2. A concentração e o tempo de imersão, para as soluções osmótica de sacarose e xarope de milho, foram os fatores de maior efeito sobre a perda de peso. A manga desidratada em solução de sacarose apresentou maiores valores de perda de peso que em solução de xarope de milho, dentro da faixa estudada.

3. A concentração e a temperatura foram os fatores que mais influenciaram a perda de água durante o processo de desidratação osmótica de manga em solução de sacarose enquanto o tempo foi o fator de maior influência, quando utilizado o xarope de milho como agente osmótico.

4. O ganho de sólidos no processo de desidratação osmótica de manga foi menor para as amostras desidratadas em solução de xarope de milho.

5. Os modelos experimentais ajustados para perda de peso, perda de água e ganho de sólidos na desidratação com sacarose, foram estatisticamente significativos, com exceção para ganho de sólidos na desidratação em solução de xarope de milho.

\section{LITERATURA CITADA}

Aguirre, J.M. de; Gasparino Filho, J. Desidratação de frutas e hortaliças. Campinas: Instituto de Tecnologia de Alimentos - ITAL. 2001. 8p. Manual Técnico

Alves, S.M.; Silveira, A.M. Estudo da secagem de tomates desidratados e não desidratados osmoticamente. Revista Universidade Rural, Rio de Janeiro, v.21, n.1, p.21-30, 2002.

Argaiz, A.; Malo, A.L.; Palou, E.; Welti, J. Osmotic dehydration of papaya with corn syrup solids. Drying Technology, New York, v.12,n.7, p.1709-1725, 1994.

Azoubel, P.M. Influência de pré-tratamento na obtenção de produtos secos de caju (anacardium occidentale L.). Campinas: UNICAMP. 2002. 154p. Tese Doutorado

Barbosa Júnior, J.L. Avaliação da influência da temperatura e concentração durante a desidratação osmótica de abóbora (cucurbita maxima) e cenoura (Daucus carota L.) utilizando-se metodologia de superfície de resposta. Campinas: UNICAMP. 2002. 124p. Dissertação Mestrado

Barros Neto, B.; Scarmínio, I.S.; Bruns, R.E. Como fazer experimentos-pesquisas e desenvolvimento na ciência e na indústria. Campinas: UNICAMP. 2001. 401p.

Borges, S.V.; Menegalli, F.C. Influência da desidratação osmótica sobre a cinética de secagem de manga. Pesquisa Agropecuária Brasileira, Brasília, v.29, n.4, p.637-642, 1994.

Box, G.E.P.; Wetz, J. Criteria for judging adequacy of estimation by an approximate response function. University of Wisconsin Technical Report, n.9, 1973.

Contreras, J.E.; Smyrl, T.G. An evaluation of osmotic concentration of apple rings using corn syrup solids solutions. Food Research International, Canadian, v.14, n.4, p.310-314, 1981.

El-Aouar, A.A. Avaliação do processo combinado de desidratação osmótica e secagem na qualidade de cubos de mamão formosa (Carica papaya L.) Campinas: UNICAMP. 2001. 113p. Dissertação Mestrado

Farsas, D.F.; Lazar, M.E. Osmotic dehydration of apples pieces: effect of temperature and syrup concentration on rates. Food Research International, Ohio, v.26, p.59-68, 1993.

Heng, W.; Guilbert, S.; Cuq, J.L. Osmotic dehydration of papaya: influence of process variables on the quality. Sciences des Aliments, Bordeaux, v.10, p.831-848, 1990.

Lomauro, C.J.; Bakshi, A.S., Labuza, T.P. Moisture transfer properties of dry and semimoist food. Journal of Food Science, Chicago, v.50, p.397-400, 1985.

Magalhães, A.M. de; Queiroz, M.R. de. Desidratação osmótica de abacaxi. In: Congresso de Iniciação Científica da UNICAMP, 9, 2001, Campinas: UNICAMP. Anais... 2001. CD Rom.

Moy, J.K.; Lau, B.H.; Dollar, A.M. Effects of sucrose and acid on osmotic-dehydration of tropical fruits. Journal of Food Processing and Preservation, Trumbull, v.2, p.131-135, 1978.

Pina, M.G.M.; Maia, G.A.; Souza Filho, M.S.M.; Figueiredo, R.W.E; Monteiro, J.C.S. Processamento e conservação de manga por métodos combinados. Revista Brasileira de Fruticultura, Jaboticabal, v.25, n.1, p.63-66, 2003.

Raoult-Wack, A.L.; Lenart, A.; Guilbert, S. Recent advances in the osmotic dehydration of foods. Trends in Food Science \& Technology, Cambridge v.5, n.8, p.225-260, 1994. 
Rastogi, N.K.; Raghavarao, K.S.M.S. Effect of temperature and concentration on osmotic dehydration of coconut. Lebensmittel-Wissenschaft \& Technologie, Oxford, v.27, n.6, p.564-567, 1994.

Santos, C.N.P. Elaboração de um estruturado de polpa de manga (Mangifera indica L. cv Tommy Atkins) parcialmente desidratada por osmose. Campinas: UNICAMP, 2003. 75p. Dissertação Mestrado
Silveira, E.T.F.; Rahman, M.S.; Buckle, K.A. Osmotic dehydration of pineapple: kinetics and product quality. Food Research International, Canadian, v.29, n.3, p.227-233, 1996.

Tedjo, W.; Taiwo, Kehinde A.; Eshtiaghi, M.N.; Knorr, D. Comparison of pretreatment methods on water and solid diffusion kinetics of osmotically dehydrated mangos. Journal of Food Engineering, New York, v.53, n.2, p.133-142, 2002. 